\title{
Níveis insuficientes de atividade física de adolescentes associados a fatores sociodemográficos, ambientais e escolares
}

\author{
Insufficient levels of physical activity of adolescents associated \\ with sociodemographic, environmental and school factors
}

\author{
Juliana da Silva ${ }^{1}$ \\ Alexandro Andrade ${ }^{1}$ \\ Renata Capistrano ${ }^{1}$ \\ Tailine Lisboa ${ }^{1}$ \\ Rubian Diego Andrade ${ }^{1}$ \\ Érico Pereira Gomes Felden ${ }^{1}$ \\ Thais Silva Beltrame ${ }^{1}$
}

${ }^{1}$ Centro de Ciências

da Saúde e do Esporte, Universidade do Estado de Santa Catarina. R. Paschoal Simoni 358, Coqueiros. 88080-350 Florianópolis SC Brasil.

julianaef@gmail.com

\begin{abstract}
This study investigated the associations between the level of physical activity with sociodemographic, environmental and academic factors of adolescents. Two thousand five hundred and forty-five adolescents aged between 14 and 18 from Florianópolis and neighboring cities participated in this study. The short version of the International Physical Activity Questionnaire (IPAQ) was used. The prevalence of inactive adolescents was $48.6 \%$, being higher among females (53.9\%). For girls, physical inactivity was associated with the lack of appropriate and free places for physical activity $(O R=1.3095 \%$ CI 1.05 to 1.82), with no influence of other variables. For boys who had no professional activity $(\mathrm{OR}=1.5495 \%$ CI 1.14 to 2.08), less educated mothers ( $O R=0.4195 \%$ $C I 0.27$ to 0.75), or graduate mothers $(O R=0.35$ 95\% CI 0,17-0,73), living in major cities (OR = $1.6495 \%$ CI 1.17 to 2.31) and low socioeconomic classification when compared to the average (OR $=0.6995 \%$ CI 0.49 to 0.97 ) were more prone to physical inactivity. It was observed that the socio-demographic variables were associated with the inactive behavior of boys and environmental variables were associated with the physical inactivity of girls. Among the academic factors, only having to repeat a year was associated with inactivity, and only for girls.
\end{abstract}

Key words Physical activity, Teenager, Adolescent health
Resumo Este estudo investigou as associações entre o nivel de atividade fisica com os fatores sociodemográficos, ambientais e escolares de adolescentes. Participaram 2.545 jovens de 14 a 18 anos dos municípios da Grande Florianópolis. Foi utilizada a versão curta do Questionário Internacional de Atividade Física (IPAQ). A prevalência de jovens inativos foi de $48,6 \%$, sendo maior no sexo feminino (53,9\%). Para as moças, a inatividade física esteve associada com a falta de lugares apropriados e gratuitos para a prática de atividade física $(O R=1,30$ IC95\%1,05-1,82), independente da influência das outras variáveis. Já para os rapazes, aqueles que não possuíam atividade profissional $(O R=1,54$ IC95\%1,14-2,08), mães de menor instrução (OR = 0,41 IC95\% 0,27-0,75) ou mães com ensino superior (OR $=0,35$ IC95\% 0,17$0,73)$, pertencentes a cidades de grande porte (OR $=1,64$ IC95\%1,17-2,31) e classificação socioeconômica baixa quando comparada à média (OR = 0,69 IC95\% 0,49-0,97), apresentaram maior chance de inatividade fisica. Observou-se que as variáveis sociodemográficas estiveram associadas ao comportamento inativo dos rapazes e às variáveis ambientais se associaram a inatividade fisica das moças. Já dentre os fatores escolares, apenas a reprovação escolar foi associada com inatividade, e somente para as moças.

Palavras-chave Atividade física, Adolescentes, Saúde do adolescente 


\section{Introdução}

A prática de atividade física na adolescência está relacionada à prevenção de doenças crônicas não transmissíveis, controle de pressão sanguínea, benefícios à saúde esquelética, bem como a aderência de um comportamento ativo na vida adulta ${ }^{1-3}$. Devido a isso, a adolescência é considerada um período essencial em relação à atividade física, ressaltando, assim, a necessidade de estímulos e oportunidades para que os jovens tenham níveis adequados de atividades físicas ${ }^{4}$.

Nos últimos anos índices elevados de inatividade física têm sido identificado entre os adolescentes. Um levantamento realizado em 122 países apontou que um a cada cinco adolescentes não atingem níveis suficientes de atividade física ${ }^{5}$. No Brasil, não há um consenso quanto ao percentual de inatividade física. No entanto em uma meta -análise sobre o tema, verificou-se que a prevalência de adolescentes brasileiros inativos variou de dois a $80 \%$ no sexo masculino e de 14 a $91 \%$ no feminino ${ }^{6}$. Diante da necessidade de maior consonância entre os estudos sobre o tema, observa-se a relevância de novas pesquisas que contemplem amostras representativas.

Além disso, diversos são os fatores que estão associados aos baixos níveis de atividade física entre os adolescentes. Dentre esses, pode-se destacar os fatores demográficos, idade e sexo ${ }^{4,7,8}$. Especialmente em relação a este último, os rapazes geralmente são mais ativos quando comparados às $\operatorname{moças}^{7-9}$. Ainda nesse aspecto, o tipo de cidade $^{10-12}$ e fatores como a falta de lugares para a prática de atividade física ${ }^{12}$ estão relacionadas ao comportamento inativo na adolescência.

Dentre os fatores socioeconômicos, a classe social ${ }^{13,14}$, escolaridade dos pais ${ }^{7,8} \mathrm{e}$ a situação ocupacional dos adolescentes ${ }^{15,16}$, apresentam destaque. No que tange o âmbito educacional, os fatores escolares, como a série ${ }^{17,18}$ e turno ${ }^{7,19}$ no qual o adolescente estuda são os que apresentam maior influência.

Investigações em torno da atividade física em adolescentes são essenciais para identificar os fatores que podem associar-se a tais comportamentos e assim proporcionar a elaboração de políticas públicas de educação em saúde voltada a realidade dos jovens. Diante disso, o presente estudo objetivou analisar as associações entre o nível insuficiente de atividade física com os fatores sociodemográficos, ambientais e escolares de adolescentes.

\section{Método}

\section{População e amostra}

A população foi composta por 24.353 adolescentes matriculados no ensino médio de 67 escolas públicas da região da Grande Florianópolis, de acordo com dados fornecidos pelo Ministério da Educação ${ }^{20}$. Essa região é composta pelos municípios de Águas Mornas ( $\mathrm{N}$ de escolas $=2 ; \mathrm{N}$ de adolescentes $=241) ;$ Angelina $(\mathrm{N}$ de escolas $=2$, $\mathrm{N}$ de adolescentes $=221$ ); Anitápolis ( $\mathrm{N}$ de escolas $=1, \mathrm{~N}$ de adolescentes $=88$ ); Antônio Carlos $(\mathrm{N}$ escolas $=1, \mathrm{~N}$ adolescentes $=390)$, Biguaçu $(\mathrm{N}$ escolas $=5, \mathrm{~N}$ adolescentes $=3396)$, Florianópolis $(\mathrm{N}$ escolas $=27, \mathrm{~N}$ adolescentes $=9813)$, Governador Celso Ramos ( $\mathrm{N}$ escolas $=2, \mathrm{~N}$ adolescentes $=406)$, Palhoça $(\mathrm{N}$ escolas $=10, \mathrm{~N}$ adolescentes $=4035)$, Rancho Queimado ( $\mathrm{N}$ de escolas $=1 ; \mathrm{N}$ de adolescentes $=107)$, Santo Amaro da Imperatriz $(\mathrm{N}$ escolas $=3, \mathrm{~N}$ adolescentes $=$ 843), São José ( $\mathrm{N}$ de escolas $=11 ; \mathrm{N}$ de adolescentes $=4604)$; São Bonifácio ( $\mathrm{N}$ de escolas $=1$, $\mathrm{N}$ de adolescentes $=90$ ) e São Pedro de Alcântara $(\mathrm{N}$ escolas $=1, \mathrm{~N}$ adolescentes $=119)($ Figura 1$)$.

Fizeram parte da população do estudo os adolescentes, com idades a partir de 14 anos, até aqueles com 18 anos completos, matriculados no ensino médio, de escolas públicas estaduais pertencentes à região da Grande Florianópolis.

A amostragem foi realizada de forma aleatória e proporcional, por conglomerados, de acordo com número de alunos matriculados nas escolas públicas e estaduais em cada município, e com base na fórmula proposta por Luiz e Magnani ${ }^{21}$. Para calcular o tamanho da amostra foi admitido um erro amostral de 3\%, nível de confiança de $95 \%$, percentual mínimo de $50 \%$, e efeito do desenho de 2,0 . O que tornou necessário selecionar uma amostra de aproximadamente 2048 estudantes. Somou-se a este valor um percentual de $50 \%$, visando a prevenção de possíveis perdas amostrais por questionários mal preenchidos e desistências (n esperado de 3072). Houve a possibilidade de aumentar o tamanho da amostra em algumas escolas, sendo possível extrapolar o $\mathrm{n}$ amostral, chegando-se a um total de 3700 questionários. Dentre esses foram excluídos aqueles que não responderam corretamente o questionário, o que resultou uma amostra de 2.545 adolescentes de escolas da rede pública estadual. Cabe destacar, que a perda amostral elevada foi por conta do processo criterioso adotado pelos avaliadores quanto ao preenchimento do questionário. Destaca-se que essas perdas amos- 

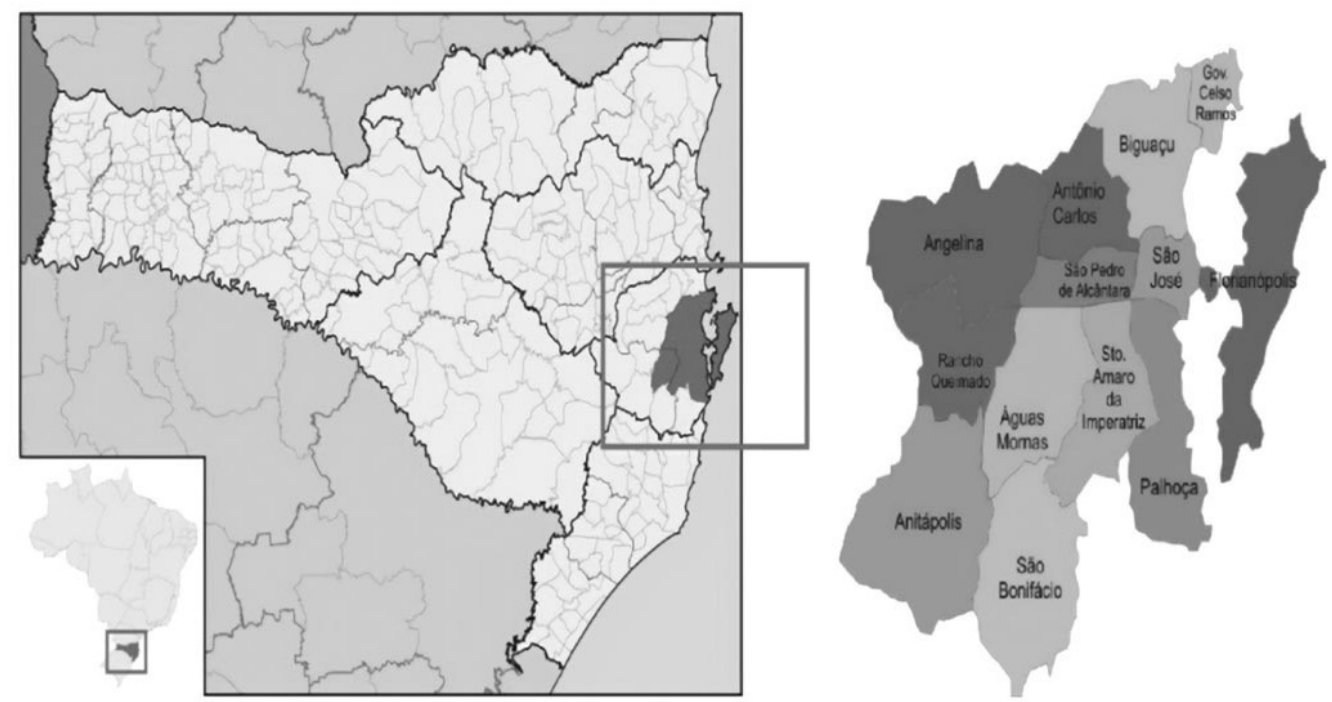

Figura 1. Mapa político da Região da Grande Florianópolis.

trais foram consideradas no plano amostral, evitando assim que o valor mínimo da amostra fosse insuficiente.

Em um primeiro momento foi realizado o sorteio das escolas participantes. Nos municípios onde existia apenas uma escola estadual com ensino médio, estas automaticamente fizeram parte da amostra. Desta forma, participaram do estudo 26 escolas, das quais 20 foram selecionadas por sorteio e seis automaticamente recrutadas por serem as únicas em seus municípios. O sorteio das turmas seguiu o mesmo critério das escolas. Nas instituições em que houvesse apenas uma turma de cada ano escolar, todas fariam parte automaticamente da pesquisa. A coleta dos dados foi realizada com 126 turmas, de primeiros, segundos e terceiros anos, nos períodos matutino, vespertino, integral (matutino e vespertino) e noturno no segundo semestre de 2013. Os escolares sorteados e autorizados para a participação na pesquisa responderam aos questionários em suas salas de aula, no turno em que estudavam acompanhados pelos pesquisadores.

\section{Instrumentos}

Para classificação do nível de atividade física utilizou-se a versão 8 , em formato curto, do Questionário Internacional de Atividade Física
(IPAQ), o qual foi validado para a população brasileira $^{22}$ e amplamente utilizado em estudos epidemiológicos ${ }^{23,24}$. Neste, o participante responde a respeito do tempo gasto em atividades físicas vigorosas, moderadas e caminhadas durante os últimos sete dias. No entanto, foram considerados apenas os minutos de atividades físicas relacionadas à intensidade moderada e vigorosa. A partir disso, foram considerados ativos os adolescentes com 300 minutos ou mais (min./semana $\geq 300$ ); e insuficientemente ativos àqueles com menos de 300 minutos semanais (min./semana $<300)^{25}$.

Para a caracterização da faixa etária os adolescentes informaram a idade, a qual foi dividida em duas faixas etárias, de 14 a 16 anos e 17 a 18 anos. Para a variável porte da cidade, a categorização utilizada levou em consideração o número de habitantes, sendo que as cidades pertencentes a este estudo foram classificadas em Grande Porte, com número de habitantes superior a 60.000 (Florianópolis, Palhoça, São José e Biguaçu) e Pequeno Porte, cujo número de habitantes fosse inferior a 25.000 (Águas Mornas, Angelina, Anitápolis, Antônio Carlos, Rancho Queimado, Santo Amaro, São Bonifácio, e São Pedro de Alcântara ${ }^{26}$.

O nível socioeconômico foi avaliado pelo questionário de classificação socioeconômica da Associação Brasileira de Empresas de Pesqui- 
sa $-\mathrm{ABEP}^{27}$ amplamente utilizado em pesquisas no Brasil. Este inventário leva em consideração a escolaridade do chefe da família e alguns bens de consumo, estratificados em oito níveis (A1, A2, B1, B2, C1, C2, D e E). Para fins de análise, a categorização foi realizada em três estratos: nível alto (A1 e A2); nível médio (B1 e B2) e nível baixo (C1, C2, D e E).

Além disso, os adolescentes foram questionados se exerciam atividades profissionais, por meio de perguntas com resposta dicotômicas (sim; não). A escolaridade dos pais e mães foi questionada com base nas classificações sugeridas pela $A B E P$, sendo agrupadas da seguinte forma: analfabeto ou séries iniciais do ensino fundamental incompletas; fundamental completo ao ensino médio completo; e ensino superior completo. As variáveis escolares foram relacionadas à série $\left(1^{\circ \mathrm{s}}, 2^{\mathrm{os}}\right.$ e $3^{\mathrm{os}}$ anos), turno de estudo (matutino, vespertino, integral e noturno) e reprovação, coletada a partir de dados do histórico escolar informado pela secretária de cada unidade de ensino.

Por fim, os adolescentes responderam de forma dicotômica (sim/não) as questões: "Você acha seguro o local onde mora?" e "No local onde você mora existem locais gratuitos e apropriados para a prática de atividades físicas, como praças, parques e quadras?", relacionadas à percepção do ambiente onde moram.

\section{Procedimentos gerais}

A pesquisa foi aprovada pelo Comitê de Ética em Pesquisas com Seres Humanos da Universidade do Estado de Santa Catarina - UDESC. A coleta iniciou após a assinatura do termo de consentimento livre e esclarecido -TCLE, pelos pais, para os menores de 18 anos, e pelos participantes do estudo.

Os dados foram coletados nas escolas e turmas previamente selecionadas para o estudo, por profissionais capacitados. O processo de preenchimento dos questionários levou aproximadamente 40 minutos.

\section{Análise estatística dos dados}

Os dados foram analisados no software Statistical Package for the Social Sciences, versão 20.0,for Windows. A normalidade da distribuição dos dados foi analisada por meio do teste de Kolmogoro$v$-Smirnov. A amostra foi caracterizada por meio de estatística descritiva, com médias, frequências e desvios-padrões. Nas análises inferenciais recorreu-se ao teste de Qui-quadrado $\left(\mathrm{X}^{2}\right)$ (de heterogeneidade ou para tendência linear) para a associação entre as variáveis categóricas. Para a diferença entre variáveis contínuas utilizou-se o teste U de Mann-Whitney.

A Regressão Logística foi utilizada para verificar possíveis associações entre os níveis insuficientes de atividade física com as variáveis sociodemográficas, ambientais e escolares, uma vez que esse modelo de regressão é a técnica mais utilizada quando se deseja controlar possíveis fatores de confusão, apontando razões de chances mais estáveis ${ }^{28}$. Para fins de análise, tendo em vista as diferentes prevalências de inatividade física entre rapazes e moças optou-se em separar os resultados por sexo. Foram analisados modelos não ajustados e ajustados. Para que fossem analisadas no modelo ajustado considerou-se na análise bruta $\mathrm{p}<0,25$ no teste do Qui-quadrado ${ }^{29}$. Adotou-se, em todas as análises estatísticas nível de significância de $5 \%$.

\section{Resultados}

Participaram do estudo 2.545 adolescentes, 1.462 $(57,4 \%)$ do sexo feminino, com média de idade de 16,09 $(1,02)$ anos. Quanto ao nível de atividade física $48,6 \%$ da amostra geral, $53,9 \%$ das moças e $41,4 \%$ dos rapazes foram considerados inativos.

Em relação às variáveis escolares a maioria da amostra estudava no turno matutino $(41,4 \%)$ e eram estudantes do primeiro ano $(39,6 \%)$. Referente à reprovação escolar, observou-se associação entre os sexos $(p=0,006)$, sendo que os rapazes apresentaram maior frequência de reprovação no histórico escolar (30,9\%), quando comparados às moças $(25,9 \%)$.

A maior parte $(71,7 \%)$ desses escolares residiam em cidades de grande porte e $85,7 \%$ relataram achar seguro o local onde moravam. Quanto aos espaços à prática de atividades físicas, $64,7 \%$ dos adolescentes do sexo masculino responderam que havia espaços apropriados e gratuitos próximo de suas residências. Já para o sexo feminino, essa percepção foi menor $(59,1 \%)$, apontando diferenças entre os sexos, quanto à percepção de locais apropriados à prática $(\mathrm{p}=0,004)$.

Quanto à escolaridade do pai e da mãe, a maior parte relatou que seus pais possuíam o ensino médio completo $(78,4 \%$ e $77,3 \%$ respectivamente). No entanto, entre os rapazes, $15 \%$ declararam que suas mães possuíam o ensino superior, enquanto que entre as moças este percentual foi 
de 11,2\%. Na classificação socioeconômica, embora a maioria dos escolares fossem de classe média (63,4\% feminino e 69,8\% masculino), 31,4\% das moças e $22,4 \%$ dos rapazes foram classificados como classe baixa. Verificou-se ainda que $40,8 \%$ dos rapazes exercem uma atividade profissional, enquanto que apenas $28,8 \%$ das moças trabalham ( $\mathrm{p}<0,001)$ (Tabela 1$)$.

Ao verificar a associação entre inatividade física com as variáveis sociodemográficas e ambientais para o ao sexo feminino, na análise não ajustada, foi identificada associação com o tipo de cidade (OR = 1,37; IC95\% 1,09-1,72) e percepção de lugares públicos adequados e gratuitos próximo de onde residem ( $\mathrm{OR}=1,25$; IC95\% $1,01-1,54)$. Observou-se que $56,2 \%$ das moças das cidades de grande porte são inativas, enquanto $48,4 \%$ das que residem em cidades de pequeno porte tem níveis insuficientes de atividade física. Referente à percepção de lugares para a prática de atividade física, 57,1\% das moças que acreditam não ter essas instalações foram identificadas como inativas fisicamente (Tabela 2).

Esses valores foram confirmados na análise ajustada, mostrando que a percepção de lugares apropriados para a prática de atividade física esteve associada a inatividade física $(\mathrm{OR}=1,30$ IC95\% 1,03-1,65). Os dados apresentados indicam que moças que não percebem esses lugares próximos de suas casas têm 1,30 mais chance de serem inativas fisicamente quando comparadas com as que percebem residir em locais com adequada estrutura à prática de atividade física (Tabela 2).

A variável reprovação escolar, na análise ajustada, esteve associada com inatividade física $(\mathrm{OR}=1,38 \mathrm{IC} 95 \%$ 1,05-1,82), sendo que as adolescentes, com ao menos uma reprovação no histórico escolar tem 1,38 mais chance de serem inativas quando comparadas as que nunca reprovaram (Tabela 2).

No sexo masculino a inatividade física esteve associada, na análise não ajustada, a atividade profissional (OR = 1,46 IC95\% 1,13-1,88), grau de instrução da mãe (OR = 0,52 IC95\% 0,330,83; OR = 0,54 IC95\% 0,32-0,93), classificação socioeconômica média $(\mathrm{OR}=0,64$ IC95\% 0,46$0,89)$, tipo de cidade $(\mathrm{OR}=1,37$ IC95\% 1,041,81 ) e percepção de segurança onde mora (OR = 1,53 IC95\% 1,08-2,16).

$\mathrm{Na}$ análise ajustada, a inatividade física foi associada com atividade profissional $(\mathrm{OR}=1,54$ IC95\% 1,14-2,08), instrução da mãe (OR = 0,45 IC95\% 0,27-0,75; OR = 0,35 IC95\% 0,17-0,73), tipo de cidade $(\mathrm{OR}=1,64$ IC95\% 1,17-2,31) e classificação socioeconômica entre a baixa e média (OR = 0,69 IC95\% 0,49-0,97). Desta forma, os dados indicam que rapazes, sem atividade profissional têm 53\% a mais de chance de serem inativos quando comparados aos que trabalham. Os jovens de cidades de grande porte têm $64 \%$ mais chances de terem níveis insuficientes de atividade física ao se compararem com adolescentes de cidades menores.

O grau de instrução da mãe e o nível socioeconômico atuaram como fator de proteção para a prática de atividade física, uma vez que os rapazes cujas mães completaram o ensino fundamental e/ou médio completo (55\%) ou o ensino superior $(66 \%)$ apresentaram menos chances de serem inativos, quando comparados aos adolescentes em que suas mães são analfabetas ou com ensino fundamental I incompleto. Referente à classe econômica, os adolescentes da classe média apresentaram 32\% menos chances de terem níveis insuficientes de atividade física em comparação aos de classe baixa (Tabela 3 ).

\section{Discussão}

O objetivo deste estudo foi analisar as associações entre o nível de atividade física com os fatores sociodemográficos, ambientais e escolares de adolescentes. A prevalência de níveis insuficientes de atividade física (48\%) foi semelhante a alguns estudos realizados no Brasil ${ }^{7,13}$. No entanto, observa-se a falta de concordância entre a maioria dos estudos quanto à prevalência de inatividade física em adolescentes brasileiros, sendo que essa pode variar de 5,4\% até 91\% $\%^{6}$. Acredita-se que tais divergências encontradas na literatura se devem ao perfil de cada tipo de população, e ainda, a utilização de diferentes instrumentos ou pontos de corte. É relevante a atenção a estes índices, tendo em vista a importância do desenvolvimento de hábitos de vida saudáveis entre os jovens, visando a sua manutenção na idade adulta ${ }^{4}$.

Ainda nesse aspecto, foi evidenciado níveis superiores de inatividade física no sexo feminino, uma vez que quase 53\% das moças foram consideradas insuficientemente ativas. Esta prevalência superior de inatividade física nas moças tem sido relatada em estudos conduzidos em outros países como, Japão ${ }^{30}$, Espanha ${ }^{31}$, Irã ${ }^{3}$ e também no Brasil ${ }^{7,8,32}$. Além dos aspectos biológicos que tornam rapazes e moças com comportamentos diferentes, essas prevalências superiores das moças inativas podem também ser explicadas por questões socioculturais, comportamentais e psi- 
Tabela 1. Descrição geral da amostra.

\begin{tabular}{|c|c|c|c|c|}
\hline Variáveis n (\%) & Geral & Feminino & Masculino & p-valor \\
\hline \multicolumn{5}{|l|}{ Atividade física } \\
\hline Inativo & $1236(48,6)$ & $788(53,9)$ & $448(41,4)$ & \multirow[t]{2}{*}{$<0,001$} \\
\hline Ativo & $1309(51,5)$ & $674(46,1)$ & $635(58,6)$ & \\
\hline \multicolumn{5}{|l|}{ Série } \\
\hline Primeiro & $997(39,6)$ & $579(49,6)$ & $418(39,2)$ & \multirow[t]{3}{*}{0,203} \\
\hline Segundo & $870(34,5)$ & $516(35,5)$ & $354(33,2)$ & \\
\hline Terceiro & $653(25,9)$ & $358(24,6)$ & $295(27,6)$ & \\
\hline \multicolumn{5}{|l|}{ Turno } \\
\hline Matutino & $1046(41,4)$ & $592(40,8)$ & $454(42,1)$ & \multirow[t]{4}{*}{0,742} \\
\hline Vespertino & $718(28,4)$ & $412(28,4)$ & $306(28,4)$ & \\
\hline Integral & $125(4,9)$ & $69(4,8)$ & $58(5,2)$ & \\
\hline Noturno & $640(25,1)$ & $378(26,1)$ & $262(24,3)$ & \\
\hline \multicolumn{5}{|l|}{ Reprovação escolar } \\
\hline Não & $1823(72,0)$ & $1079(74,1)$ & $744(69,1)$ & \multirow[t]{2}{*}{0,006} \\
\hline Sim & $709(28,0)$ & $377(25,9)$ & $332(30,9)$ & \\
\hline \multicolumn{5}{|l|}{ Faixa etária } \\
\hline $14-15-16$ & $1477(64,1)$ & $892(66,7)$ & $585(60,6)$ & \multirow[t]{2}{*}{0,003} \\
\hline $17-18$ & $82(35,9)$ & $446(33,3)$ & $381(39,4)$ & \\
\hline \multicolumn{5}{|l|}{ Atividade Profissional } \\
\hline Não trabalha & $1641(66,1)$ & $1019(71,2)$ & $622(59,2)$ & \multirow[t]{2}{*}{$<0,001$} \\
\hline Trabalha & $841(33,9)$ & $413(28,8)$ & $428(40,8)$ & \\
\hline \multicolumn{5}{|l|}{ Grau de Instrução Pai } \\
\hline Analfabeto/ fundamental 1 incompleto & $330(13,5)$ & $196(13,4)$ & $134(12,8)$ & \multirow[t]{3}{*}{0,272} \\
\hline Fundamental 1 completo/médio completo & $1912(78,4)$ & $1096(78,6)$ & $816(78,2)$ & \\
\hline Superior & $197(8,1)$ & $103(7,4)$ & $94(9,0)$ & \\
\hline \multicolumn{5}{|l|}{ Grau de Instrução Mãe } \\
\hline Analfabeto/ fundamental 1 incompleto & $246(9,9)$ & $162(11,3)$ & $84(7,9)$ & \multirow[t]{3}{*}{0,001} \\
\hline Fundamental 1 completo/médio completo & $1929(77,3)$ & $1113(77,5)$ & $816(77,1)$ & \\
\hline Superior & $320(12,8)$ & $161(11,2)$ & $159(15,0)$ & \\
\hline \multicolumn{5}{|l|}{ Tipo de cidade } \\
\hline Pequeno porte & $719(28,3)$ & $426(29,1)$ & $293(272)$ & \multirow[t]{2}{*}{0,248} \\
\hline Grande porte & $1826(71,7)$ & $1036(70,9)$ & $890(72,9)$ & \\
\hline \multicolumn{5}{|l|}{ Classificação socioeconômica } \\
\hline Baixa & $583(27,6)$ & $382(31,4)$ & $201(22,4)$ & \multirow[t]{3}{*}{$<0,001$} \\
\hline Média & $1399(66,1)$ & $772(63,4)$ & $627(69,8)$ & \\
\hline Alta & $134(6,3)$ & $64(5,3)$ & $70(7,8)$ & \\
\hline \multicolumn{5}{|l|}{ Com quem mora } \\
\hline Mãe e pai & $1751(69,1)$ & $995(68,1)$ & $756(70,1)$ & \multirow[t]{4}{*}{0,007} \\
\hline Somente Mãe & $537(21,2)$ & $327(22,4)$ & $210(19,5)$ & \\
\hline Somente Pai & $95(3,7)$ & $41(2,8)$ & $54(5,0)$ & \\
\hline Não mora com os pais & $152(6,0)$ & $94(6,5)$ & $58(5,4)$ & \\
\hline \multicolumn{5}{|l|}{ Acha seguro onde mora? } \\
\hline Não & $362(14,3)$ & $215(14,8)$ & $147(13,6)$ & 0,423 \\
\hline Sim & $2170(85,7)$ & $1240(85,2)$ & $930(86,4)$ & \\
\hline $\begin{array}{l}\text { Lugares gratuitos e apropriados para a prática } \\
\text { física }\end{array}$ & & & & \\
\hline Não & $974(38,5)$ & $594(40,9)$ & $380(35,3)$ & 0,004 \\
\hline Sim & $1557(61,5)$ & $860(59,1)$ & $697(64,7)$ & \\
\hline
\end{tabular}


Tabela 2. Associação entre inatividade física e variáveis sociodemográficas, ambientais e escolares para o sexo feminino.

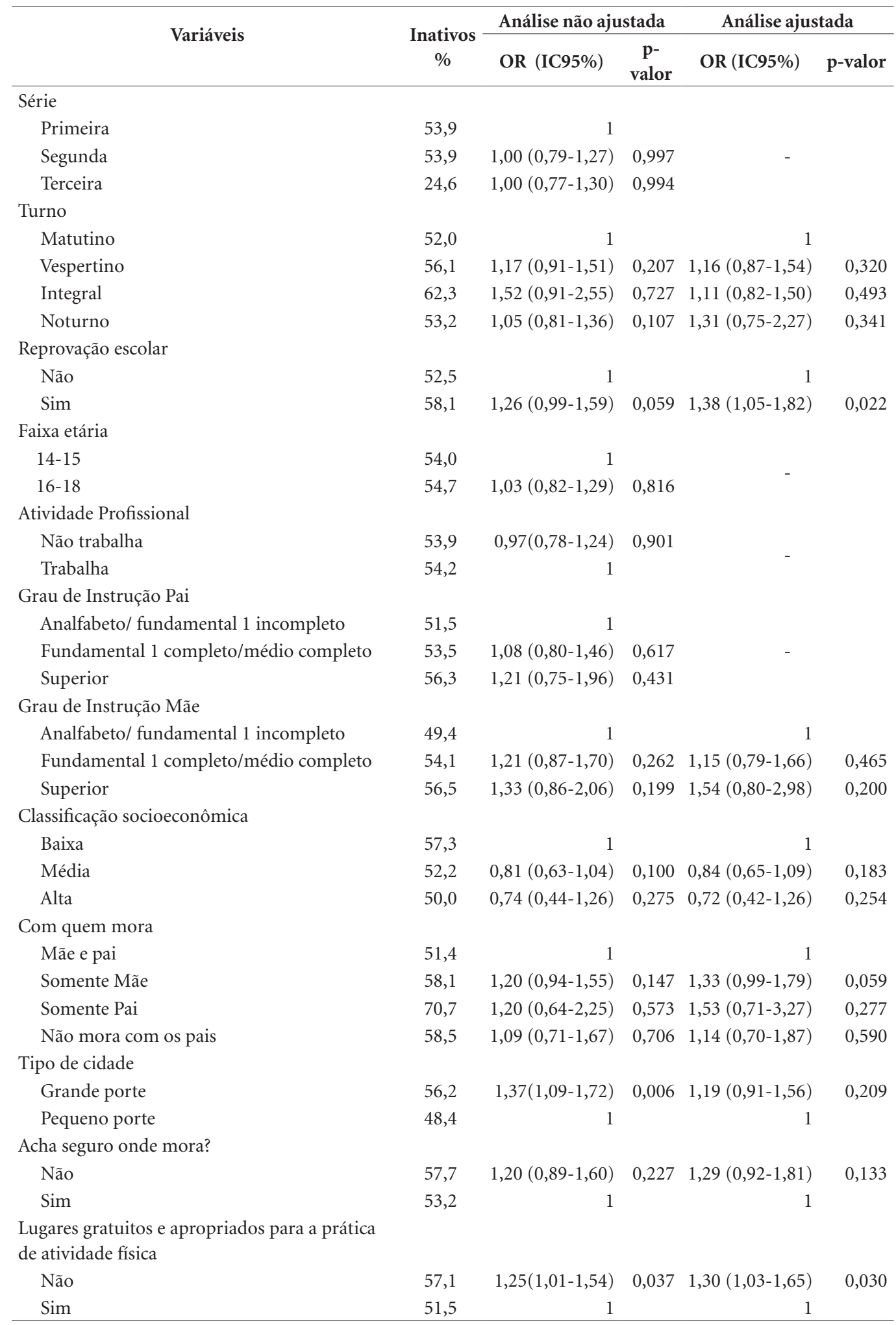

Teste de associação por meio da Regressão logística binária. IC95\% (intervalo de 95\% de confiança). 
Tabela 3. Associação entre inatividade física e variáveis sociodemográficas, ambientais e escolares para o sexo masculino.

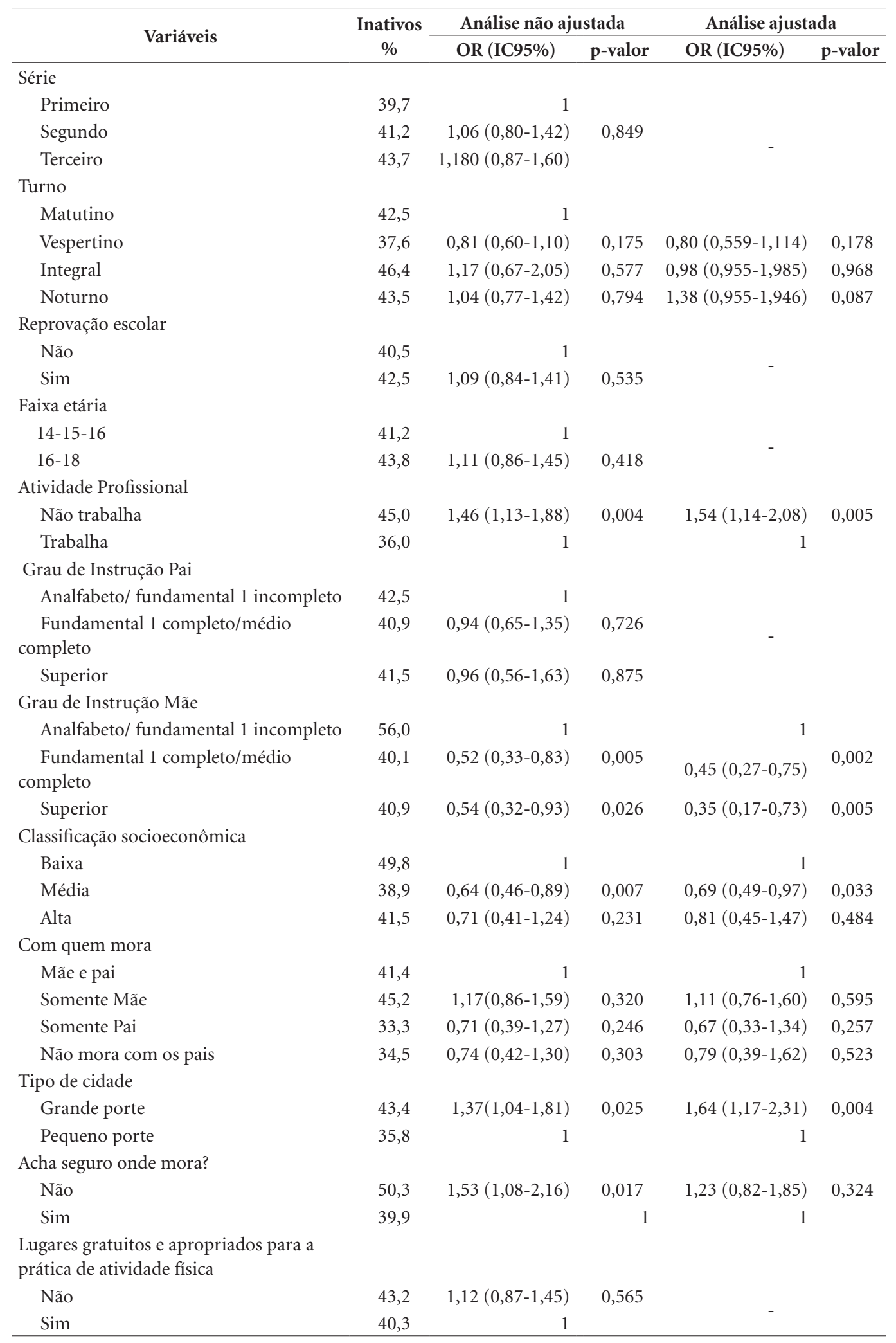

Teste de associação por meio da Regressão logística binária. IC95\% (intervalo de 95\% de confiança). 
cológicas, pois os rapazes ainda são incentivados a realizarem atividades físicas mais vigorosas que as meninas, o que pode ter influenciado nesses resultados ${ }^{33}$. É com base na relação com os amigos, familiares e sob a influência da mídia que os adolescentes constroem sua identidade e identificam o que é socialmente aceito ou não. No entanto, observa-se que estes componentes sociais ainda encorajam as moças a participarem apenas de atividades físicas leves e os rapazes atividades mais vigorosas ${ }^{7}$.

No que se refere aos fatores ambientais, estudos analisam os aspectos relacionados aos tipos de cidade e a atividade física com base nas características geográficas, como região urbana e rural ${ }^{34}$, capitais sociais ${ }^{8}$, estrutura física ${ }^{10,12}$, Índice de desenvolvimento humano - $\mathrm{IDH}^{35}$, entre outros. No presente estudo, foi utilizado o porte de cidade baseado no número de habitantes (cidades de pequeno e grande porte). A partir dessa análise, foram identificadas associações significativas entre a inatividade física e o porte de cidade para os rapazes, sendo que aqueles que residem em cidades de grande porte têm mais chance de serem inativos fisicamente quando comparados aos que moram em cidades de pequeno porte. Esse fenômeno possivelmente é decorrente a aspectos sociais e ambientais, no qual o adolescente está inserido. Devido a urbanização e o crescimento acelerado das grandes cidades, observa-se o aumento do comportamento inativo do adolescente justificado pela maior opção de atividades sedentárias ${ }^{36}$. Além disso, os facilitadores para a atividade física, como segurança, conforto, acessibilidade e confiança ao seu redor ${ }^{37}$ possivelmente são encontrados em cidade com menor número de habitantes.

Nesse contexto, em estudo recente realizado em Londres, uma cidade com alto número de habitantes, o tempo em atividade física não apresentou diferenças entre os quatro bairros investigados com prevalências de níveis insuficientes de atividades físicas em torno de $87 \%$ para as moças e $72 \%$ entre os rapazes. No entanto, os pesquisadores identificaram que os adolescentes de bairros com maior facilidade de deslocamento por bicicletas, na percepção dos adolescentes, possuíam maior tempo despendido em atividades físicas $^{38}$. Sugerindo que, a infraestrutura adequada para o deslocamento ativo possa ser um fator importante para elevar os padrões de atividade físicas em adolescentes também nas grandes cidades brasileiras ${ }^{35}$.

Além disso, os dados do presente estudo indicam que no sexo feminino houve associação entre níveis insuficientes de atividade física com a percepção de ausência de lugares apropriados e gratuitos para a prática de atividade física, sendo que moças que relataram não identificar locais para a prática tem $29 \%$ mais chances de serem inativas quando comparadas às suas opostas. Um estudo realizado na Carolina do Norte (EUA) apontou que instalações adequadas foram associadas com o comportamento ativo para o sexo feminino, assim como no presente estudo essa relação não ocorreu com os rapazes ${ }^{39}$. Isso pode ser explicado pela maior restrição às moças em relação aos espaços para a prática. Dessa forma, é necessária a disponibilidade de lugares com programação e instalações apropriadas para a prática de atividade física voltada às moças ${ }^{12,39}$. Em estudos brasileiros, a associação entre a ausência de instalações adequadas para a prática atividade física e inatividade física ocorreu independente dos sexos ${ }^{12,40}$, devido a característica de modelos de análise dos estudos.

No sexo masculino a percepção de segurança onde mora esteve associada com inatividade física, uma vez que aqueles que não se sentem seguros tem maior probabilidade de serem inativos quando comparados aos com maior percepção de segurança no lugar onde moram. A falta de segurança é entendida como uma restrição para a prática de atividade física, e esta parece exercer influência no comportamento ativo entre os adolescentes ${ }^{41,42}$.

No que diz respeito a classificação socioeconômica, apenas entre os rapazes a inatividade física apresentou associação. Sendo que aqueles de classe média têm menos chances de ser inativos quando comparados aos de classe baixa. Assim como em um estudo realizado em Macapá-AP, o qual indicou que os rapazes de classe $\mathrm{C}$ têm duas vezes mais chances de serem inativos quando comparado aos de classes superiores ${ }^{23}$. Em outro contexto, em Curitiba, região sul do país, essa associação não ocorreu com o sexo mascu$\operatorname{lino}^{43}$. Destaca-se que no presente estudo não foi identificado associação entre inatividade física e classe social alta quando comparado a classe baixa. Nessa perspectiva, entende-se que ter acesso à prática de atividade física e de esportes relacionase com o fato da família ter condições financeiras para ofertar o acesso às práticas ${ }^{44}$.

Ainda nesse contexto, os adolescentes do sexo masculino, deste estudo, que não trabalham têm $53 \%$ mais chances de serem inativos. Isso pode ser decorrente de características das atividades laborais sem que os adolescentes estejam expostos, sendo que uma característica marcante das 
cidades de pequeno porte, participantes deste estudo são as atividades econômicas relacionadas a agricultura familiar, criação de animais, onde os adolescentes auxiliam seus pais em trabalhos do cotidiano. Características que podem influenciar de diferentes maneiras, no estilo de vida dos adolescentes e de suas famílias.

Relacionado à escolaridade dos pais, os rapazes em que as mães não são analfabetas ou semianalfabetas têm mais chances de serem inativos. Outros estudos também encontraram essa associação ${ }^{8,23}$. Uma vez que pais com maior instrução tendem a ter maior conhecimento sobre aspectos relacionados à saúde ${ }^{8,33,41}$. Essa relação com a escolaridade materna ocorre devido a figura materna atuar como fator de proteção em relação a comportamentos saudáveis de adolescentes ${ }^{45}$.

No que diz respeito às variáveis escolares, série e turno escolar, não foi identificado associação com níveis de atividade física para ambos os sexos. Relacionado ao turno, um estudo realizado no estado de Santa Catarina encontrou associação entre essa variável e inatividade física ${ }^{16}$. No entanto, foram analisados apenas turno diurno e noturno e, para o presente estudo, fizeram parte adolescentes de turno matutino, vespertino, integral e noturno. Já a variável reprovação escolar foi associada à inatividade física para o sexo feminino. Uma revisão sistemática indicou que os níveis adequados de atividade física estão associados ao rendimento acadêmico de forma positiva, sendo que crianças com melhor capacidade aeróbia apresentam um perfil neuroelétrico mais eficiente, aumento da densidade das sinapses e vascularização do córtex cerebral ${ }^{46}$.

Este estudo limitou-se a utilizar uma medida indireta para analisar o nível de atividade física. Sabe-se que o padrão ouro para estas análises é a utilização de medidas diretas como a acelorometria. No entanto, por questões de recursos mate- riais e logísticos, não foi possível inferir essa variável a partir destas medidas. Contudo, destacam-se como pontos positivos do presente estudo a utilização de uma amostra representativa que fortalece as análises e associações identificadas, dando a possibilidade de generalização e comparações dos resultados encontrados com outras populações.

\section{Conclusão}

Em relação ao nível de atividade física dos adolescentes, diferentes fatores sociodemográficos devem ser considerados. Moças e rapazes apresentaram comportamentos distintos no que se refere à prática de atividade física. De forma geral, as moças demonstraram ser mais inativas quando comparadas aos rapazes. O porte da cidade parece ter grande influência na prática regular de atividade física tanto para moças quanto para rapazes. Sendo que os adolescentes das cidades de grande porte têm maior probabilidade de apresentarem níveis insuficientes de atividade física.

Quanto aos fatores escolares série e turno parecem não ter relação com o nível de atividade física, já reprovação escolar esteve associada com inatividade física, apenas no sexo feminino.

Este trabalho vem a contribuir com os estudos referentes ao nível de atividade física em adolescentes, com uma maior compreensão das variáveis associadas ao comportamento inativo. Espera-se ainda, que a partir dos dados provenientes do presente estudo, seja possível uma reflexão sobre as políticas públicas, relacionadas à promoção da saúde na adolescência, inserindo nesse contexto não apenas os jovens, mas também sua família, e outras pessoas próximas a ele. Uma vez que o comportamento ativo mais significativo pode ser, consequentemente, mais duradouro. 


\section{Colaboradores}

J Silva, AA e TS Beltrame trabalharam na concepção, delineamento, análise e interpretação dos dados, redação do artigo, revisão crítica, e a aprovação da versão a ser publicada; R Capistrano, $T$ Lisboa, RD Andrade e EPG Felden na análise e interpretação dos dados, redação do artigo, revisão crítica, e na aprovação da versão a ser publicada.

\section{Referências}

1. Ho FKW, Louvie LHT, Chow CB, Wong WH, Ip P Physical activity improves mental health through resilience in Hong Kong Chinese adolescents. BMC Pediatrics 2015; 15(48):2-9.

2. Marques A, Calmeiro L, Loureiro N, Frasquilho D, Matos MG. Health complaints among adolescents: Associations with more screen-based behaviours and less physical activity. J Adolesc 2015; 44:150-157

3. Bélanger M, Sabiston CM, Barnett TA, O'Loughlin E, Ward S, Contreras G, O'Loughlin J. Number of years of participation in some, but not all, types of physical activity during adolescence predicts level of physical activity in adulthood: Results from a 13-year study. Int J Behav Nutr Phys Act 2015; 12(76):2-8.

4. Dumith SC, Gigante DP, Domingues MR, Hallal PC, Menezes AM, Kohl HW. Predictors of physical activity change during adolescence: a 3.5-year follow-up. Public Health Nutr 2012; 15(12):2237-2245

5. Hallal PC, Andersen LB, Bull FC, Guthold R, Haskell W, Ekelund U. Global Physical activity levels: surveillance progress, pitfalls, and propescts. Lancet 2012; 280(9838):247-257.

6. Barufaldi, LA, Abreu, GA, Coutinho, ES, Bloch, KV Meta-analysis of the prevalence of physical inactivity among Brazilian adolescents Cad Saude Publica 2012; 28(6):1019-1032.

7. Farias Júnior JC, Lopes AS, Mota J, Hallal PC. Prática de atividade física e fatores associados em adolescentes no Nordeste do Brasil. Rev Saude Publica 2012; 46(3):505-515.

8. Hallal PC, Knuth AG, Cruz DKA, Mendes MI, Malta DC. Prática de atividade física em adolescentes brasileiros. Cien Saude Colet 2010; 15(2):3035-3042.

9. Fermino RC, Rech CR, Hino AAF, Añez CRR, Reis RS. Atividade física e fatores associados em adolescentes do ensino médio de Curitiba, Brasil. Rev Saude Publica 2010; 44(6):986-995.

10. Silva IJO, Alexandre MG, Ravagnani FCP, Silva JVP, Colelho-Ravagnani CF. Atividade física: espaços e condições ambientais para sua prática em uma capital brasileira. R. Bras. Ci. e Mov. 2014; 22(3):53-62.
11. Marques RMB, Fornes NS, Stringhini MLF. Fatores socioeconômicos, demográficos, nutricionais e de atividade física no controle glicêmico de adolescentes portadores de diabetes melito tipo 1. Arq Bras Endocrinol Metab 2011; 55(3):194-202.

12. Farias Júnior JC, Reis RS, Hallal PC. Atividade física e fatores psicossociaais ambientais em adolescentes do Nordeste o Brasil. Cad Saude Publica 2014; 30(5):941951.

13. Alves CFA, Silva RDCR, Assis AMO, Souza CJPO, Frainer DES. Fatores associados à inatividade física em adolescentes de 10-14 anos de idade, matriculados na rede pública de ensino do município de Salvador, BA. Rev Bras Epidemiol 2012; 15(4):858-870.

14. Reichert FF, Hallal PC, Wells JC, Horta BL, Ekelund U, Menezes AM. Objectively measured physical activity in the 1993 Pelotas (Brazil) birth cohort. Med Sci Sports Exerc 2012; 44(12):2369-2375.

15. Oliveira NKR, Barros MVG, Reis RS, Tassitano RM, Tenório MCM, Bezerra J, Farias Júnior JC. Estágios de mudança de comportamento para a atividade física em adolescentes. Motriz rev. educ. Fís 2012; 18(1):42-54.

16. Farias Júnior JC, Nahas MV, Barros MVG, Loch MR, Oliveira ESA, Bem MFL, Lopes AS. Comportamentos de risco à saúde em adolescentes no Sul do Brasil: prevalência e fatores associados. Rev Panam Salud Publica 2009; 25(4):344-352.

17. Oliveira TC, Silva AAM, Santos CJN, Sousa J, Conceição SIO. Atividade física e sedentarismo em escolares da rede pública e privada de ensino em São Luís. Rev Saude Publica 2010; 44(6):996-1004.

18. Dumith SC, Domingues MR, Gigante DP, Hallal PC, Menezes AM, Kohl HW. Prevalence and correlates of physical activity among adolescents from Southern Brazil. Rev Saude Publica 2010; 44(3):457-467.

19. Viana MS, Andrade A, Matias TS. Teoria da autodeterminação: aplicação no contexto dos exercícios físicas de adolescentes. Pensar a Prática 2010; 13(2):1-8.

20. Brasil. Lei 9.394, de 20 de dezembro de 1996. Estabelece as diretrizes e bases da educação nacional. Diário oficial da União 1996; 21 dez. 
21. Luiz RR, Magnanini MMF. A lógica da determinação do tamanho da amostra em investigações epidemiológicas. Cadernos de Saúde Coletiva 2000; 8(2):9-28.

22. Guedes DP, Lopes CC, Guedes JERP. Reprodutibilidade e validade do Questionário Internacional de Atividade Física em adolescentes. Revista Brasileira de Medicina do Esporte 2005; 11(2):151-158.

23. Alberto AAD, Figueira Junior AJ. Prevalência de inatividade física e sua associação com variáveis sociodemográficas em adolescentes do Município de Macapál AP. R. bras. Ci. e Mov 2015; 23(4):80-93.

24. Pelegrine A, Silva DAS, Glaumanni GS, Cardoso TE, Silva JMFL, Petroski EL. Prática de caminhada, atividade física moderada e vigorosa e fatores associado em adolescentes de uma capital do sul do Brasil. Rev. bras. cineantropom. desempenho hum 2015; 17(1):11-20.

25. United States Department of Health and Human Services. Physical Activity Guidelines Advisory Committee Report, 2008. Washington: United States Department of Health and Human Services; 2008.

26. Instituto Brasileiro de Geografia e Estatística (IBGE). Censor Demographic 2000. Rio de Janeiro: IBGE; 2010.

27. Associação Brasileira das Empresas de Pesquisas (ABEP). Critério Padrão de Classificação Econômica Brasil/2014. [acessado 2016 Jul 7]. Disponível em: http://www.abep.org/criterio-brasil

28. Hirakata VN. Alternativas de análise para um desfecho binário em estudos transversais e longitudinais [dissertação]. Pelotas: Universidade Federal de Pelotas; 1999.

29. Hosmer JDW, Lemeshow S, Sturdivant RX, editors. Applied Logistic Regression. Hoboken: Wiley; 2013.

30. Ishii K, Shibata A, Adachi M, Nonoue K, Oka K. Gender and grade differences in objectively measured physical activity and sedentary behavior patterns among Japanese children and adolescents: a cross-sectional study. BMC Public Health 2015; 15(1):1254.

31. Mielgo-Ayuso J, Aparicio-Ugarriza R, Castillo A, Ruiz E, Ávila JM, Aranceta-Batrina J, Gil A, Ortega RM, Serra-Majem L, Varela-Moreiras G, González-Gross M. Physical Activity Patterns of the Spanish Population Are Mostly Determined by Sex and Age: Findings in the ANIBES Study. PloSone 2016; 11(2):e0149969.

32. Cureau FV, Silva TLN, Bloch KV, Fujimori E, Belfort DR, Carvalho KMB, Leon EB, Vasconcelos MTL, Ekelund U, Schaan BD. ERICA: inatividade física de lazer em adolescentes brasileiros. Rev Saude Publica 2016; 50(Supl. 1):1-11.

33. Dumith SC, Santos MN, Teixeira LO, Cazeiro CC, Mazza SE, Cezar JA. Prática de atividade física entre jovens em município do semiárido no Brasil. Cien Saude Colet 2016; 21(4):1083-1093.

34. Moore JB, Jilcott SB, Shores KA, Everson KR, Brownson RC, Novick LF. A qualitative examination of perceived barriers and facilitators of physical activity for urban and rural youth. Health Educ Res 2010; 25(5):355-367.

35. Siva DA. Relationship between Brazilian adolescents' physical activity and social and economic indicators of the cities where they live. Percept Mot Skills 2015; 120(2):355-366.
36. Thielinan J, Rosella L, Copes R, Lebenbaum M, Manson H. Neighborhood walkability: Differential associations with self-reported transport walking and leisure-time physical activity in Canadian towns and cities of all sizes. Prev Med 2015; 77:174-180

37. Smith AL, Troped PJ, McDonough MH, DeFreese JD. Youth perceptions of how neighborhood physical environment and pers affect physical activity: a focus group study. International Jornal of Behavirl Nutrition and Physical Activity. 2015; 12(80):1-9.

38. Smith NR, Lewis DJ, Fahy A, Eldridge S, Taylor SJ, Moore DG, Clark C, Stansfeld SA, Cummins S. Individual socio-demographic factors and perceptions of the environment as determinants of inequalities in adolescent physical and psychological health: the Olympic Regeneration in East London (ORiEL) study. BMC Public health 2015; 15(15):2-18.

39. Frazer A, Voss C, Winters M, Naylor PJ, Higgins JW, McKay H. Differences in adolescents' physical activity from school-travel between urban and suburban neighbourhoods in Metro Vancouver, Canada. Prev Med Rep 2015; 2:170-173

40. Copettii J, Neutzling MB, Silva MC. Brarreiras à pratica de atividades físicas em adolescentes de uuma cidade do sul do Brasil. Rev, Bras. Ativ. Fís. Saúde. 2010; 13(2):88-94.

41. Santos MS, Fermino RC, Reis RS, Cassou AC, Añes CRR. Barreiras para a prática de atividade física em adolescentes. Um estudopor grupos focais. Rev. bras. cineantropom. desempenho hum 2010; 12(3):137-147.

42. Dwywe JJ, Alison KR, Goldenberg ER, Fein AJ, Yoshida KK, Boutilier MA. Adolescente girls' perceived barriers to participation in physica activity. Adolescentey. Adolescence 2006; 41(161):75-89.

43. Santos GC, Neto AS, Sena JS, Campos W. Atividade física em adolescentes: uma comparação entre os sexos, faixas etárias e classes econômicas, Rev Bras Ativ Fis Saúde 2014; 19(4):455-464.

44. Eime RM, Charity MJ, Harvey JT, Payne WR. Participation in Sport and physical activity: associations with sócio-economic status and geographical remoteness. BMC Public Health 2015; 15(343):1-12.

45. Intituto brasilero de geografia e estátistica (IBGE). Pesquisa nacional de saúde do escolar. Rio de Janeiro: IBGE; 2012.

46. Peralta M, Mauricio I, Lopes M, Costa S, Sarmento H, Marques A. Relação entre educação física e rendimento acadêmico do adolescentes: uma revisão sistemática. Rev de Psicologia da Criança e do Adolesc 2014; 5(2):129-137.

Artigo apresentado em 31/07/2016

Aprovado em 09/12/2016

Versão final apresentada em 11/12/2016 